\title{
Correlación neuropsicológica y electrofisiológica en niños escolares con TCE
} Neuropsychological and electrophysiological correlation in children with TCE

\author{
Abel Delgado ${ }^{1}$, Luis Quintanar ${ }^{1 *}$, Yulia Solovieva ${ }^{1}$, Regina Machinskaya ${ }^{2}$ \\ 1 Facultad de Psicología, Universidad Autónoma de Puebla. Puebla, México. \\ 2 Laboratorio de Neurofisiología de los Procesos Cognitivos, Instituto de Fisiología del Desarrollo, Academia de Educación, Moscú, Rusia.
}

\section{Resumen}

El traumatismo craneoencefálico (TCE) es una de las causas principales por la que se solicita atención médica en el servicio hospitalario de urgencias. El TCE frecuentemente produce daño cerebral o deterioro funcional del sistema nervioso en distintos niveles, que se refleja en las funciones cognitivas, tanto en niños como en adultos. El objetivo del presente estudio es correlacionar los resultados obtenidos a través de la Tomografía Axial Computarizada (TAC), el registro electroencefalográfico (EEG) y la evaluación neuropsicológica en niños escolares con TCE. En el estudio participaron ocho niños de 5 a 8 años de edad, diagnosticados con TCE leve, moderado y severo. Todos fueron evaluados con la prueba "Diagnóstico neuropsicológico breve Infantil Puebla-Sevilla" y se les realizó el registro EEG. Los datos clínicos y electrofisiológicos se compararon con la información obtenida por la TAC. Los resultados del estudio muestran una alta correlación entre el análisis cualitativo visual del EEG y de la evaluación clínica neuropsicológica, y una baja correlación de estos con los datos de la TAC. Se sugiere que la combinación de la evaluación neuropsicológica y el registro electrofisiológico tiene un mayor nivel predictivo acerca del cuadro clínico que resulta como consecuencia de un TCE en niños escolares.

Palabras clave: traumatismo craneoencefálico en niños, evaluación neuropsicológica, análisis cualitativo de EEG

\begin{abstract}
TCE is one of the most frequent causes of medical urgent attention. Both in adults and children TCE produces brain damage with functional changes in nervous system on its different levels, which are reflected in cognitive behavior both in children and adults. The goal of this study is to establish possible correlations between results obtained by psychophysiological methods (TAC and EEG) and neuropsychological assessment of children with TCE. 8 children between 5 and 8 years old were included in the study. The children received medical diagnostic of light, moderate or severe TCE given by Medical Urgency Service. All children were evaluated by "Neuropsychological assessment for children Puebla-Sevilla and by EEG register. The procedure of qualitative visual analysis of EEG results was carried out. The obtained results were contrasted with the TAC data. The results of comparison pointed out high correlation between EEG analysis and neuropsychological syndromes and less significant correlation between TAC and neuropsychological assessment. We suggest effective predictive possibility of combination of clinical, neuropsychological and neurophysiological methods of analysis in cases of TCE in children.
\end{abstract}

Keywords: TCE in children, neuropsychological assessment, qualitative analyses of EEG

\footnotetext{
* Correspondencia: luis.quintanar@correo.buap.mx. Facultad de Psicología, Universidad Autónoma de Puebla, 3 Oriente 403, Centro, Puebla, 72000, México. Teléfono: +52 (222) 2425370

Recibido: 12-08-11. Revisión desde: 12-09-11. Aceptado: 12-10-11

DOI: $10.5839 / \mathrm{rcnp} .2011 .0602 .07$
} 


\section{Introducción}

El traumatismo craneoencefálico (TCE) frecuentemente produce lesión o deterioro funcional del sistema nervioso (Casas, 2008; Stocchetti, 2010) y constituye una de las principales causas de muerte en la mayoría de los países en desarrollo. En México, el TCE ocupa el tercer lugar después de los problemas cardiovasculares y el cáncer (Secretaría de Salud, 2008) y es la primera causa de muerte y discapacidad infantil. Se estima que cerca del $75 \%$ de los ingresos hospitalarios por trauma involucran lesión craneal y en el 50\% de los casos de niños que presentan politraumatismos, comprometiendo la supervivencia de los pacientes en aproximadamente un 70 a $80 \%$ de los casos (Gómez, 2004).

En Estados Unidos, el TCE es la principal causa de muerte y discapacida $d$ en personas menores de 45 años, con una incidencia de 180 a 250 por cada 100.000 habitantes (Bruns, 2003), la cual incrementa en la población pediátrica hasta 200 a 500 por cada 100.000 habitantes (Anderson, 2010).

Los avances tecnológicos y médicos han logrado disminuir la tasa de mortalidad por esta causa, pero ha incrementado el número de pacientes con secuelas neurológicas y alteraciones residuales, constituyendo uno de los problemas de salud más graves de la sociedad (González, Pueyo y Serra, 2004). Pero, además de identificar la lesión, el hematoma y otros elementos a través de la tomografía axial computarizada, es necesario determinar sus secuelas neuropsicológicas (Muñóz-Céspedes, 2001).

Las alteraciones neurológicas, cognitivas y conductulaes producidas por un TCE son muy variadas y se relacionan con la localización y extensión del daño cerebral, el grado de severidad, la edad y las características premórbidas del paciente, entre otras (Bigler, 1990; Bejarano, 2008). Por ejemplo, se han reportado alteraciones de las funciones ejecutivas (Mangeot, 2002), la planeación para la solución de problemas (Jacobs, 2002), la atención, la memoria y la coordinación motora (Ewing-Cobbs, 1998), y secuelas importantes a largo plazo sobre el desarrollo y el aprendizaje, así como en la vida cotidiana.

El estudio de las alteraciones cognitivas que surgen como consecuencia de TCE en la edad infantil, a través de la evaluación neuropsicológica cualitativa, puede aportar datos acerca del grado y el tipo de alteraciones en relación con la edad psicológica del niño, y desarrollar programas interventivos para la superación de las dificultades (Quintanar y Solovieva, 2008). La cualificación de los síntomas se realiza a través de un análisis detallado de las ejecuciones de los niños y permite identificar la causa común que los produce, además de señalar sus mecanismos cerebrales (Luria, 1969; Quintanar y Solovieva, 2002, 2003, 2008).

De especial interés es la correlación de los resultados de la evaluación neuropsicológica con la localización de la lesión y las características de la actividad cerebral, a través del análisis cualitativo visual del registro electroencefalográfico (EEG), que permite la identificación de los patrones de desarrollo cerebral, tanto morfológico como de la organización funcional en redes neuronales (Machinskaya, 2006), y complementa las valoraciones clínicas neuropsicológicas (Thatcher, 2001a, Thatcher, 2001b, Pivik, 1993), ya que determina si la actividad registrada es de naturaleza cortical o subcortical (Machinskaya,
1997; Bezrukikh, 1999; Machinskaya, 2006; Mundy, 2003; Solovieva, Machinskaya y Quintanar, 2009).

Los patrones electroencefalográficos homogéneos se distribuyen en bloques de acuerdo a su significado funcional, los cuales caracterizan el estado de los sistemas corticales ritmógenos en general (bloque 1), los cambios cerebrales generales (bloque 2), el estado funcional de los sectores locales cerebrales, como presencia o ausencia de patrones desviados locales (bloque 3), y el estado funcional de las estructuras subcorticales regulatorias (bloque 4) de diversos niveles (Lukashevich, Machinskaya y Fishman, 1999).

El objetivo de este trabajo es correlacionar los efectos neuropsicológicos que se observan en niños de 5 a 8 años con TCE, con la dinámica de la actividad cerebral eléctrica obtenidos a través de un registro de EEG y con la localización del posible daño cerebral (TAC).

\section{Método}

Sujetos

En el estudio participaron cuatro niñas y cuatro niños: dos de 5 años, cuatro de 7 y dos de 8 años, los cuales sufrieron un TCE leve (1), moderado (2) y severo (5). Todos ellos fueron atendidos en la Unidad de Traumatología y Ortopedia del IMSS de Puebla (tabla 1).

Los criterios de inclusión fueron los siguientes:

- Haber sufrido un TCE abierto o cerrado, focal o difuso.

- Tener una edad de 5,0 a 8,11 años.

- Asistir a una institución preescolar o escolar regular hasta el día del TCE.

- No haber presentado problemas de aprendizaje ni trastornos neurológicos o psiquiátricos previos al TCE.

- Tener más de 1 mes y menos de 12 meses de transcurrido el evento de TCE al momento de la evaluación neuropsicológica y electrofisiológica.

Criterios de exclusión:

- Incapacidad física para la realización de las tareas de la valoración neuropsicológica.

- Imposibilidad para realizar el EEG.

\section{Material}

Para la valoración neuropsicólogica se utilizó la prueba "Evaluación neuropsicológica infantil Puebla-Sevilla" (Quintanar, Solovieva y León-Carrión, en prensa). La prueba se fundamenta en las aportaciones del modelo histórico-cultural (tabla 2) y explora el estado funcional de sectores corticales especializados de los niños (Quintanar y Solovieva, 2008; Solovieva y Quintanar, 2009).

El registro electroencefalográfico se realizó con el equipo computarizado Nicolet (Estados Unidos), con frecuencia de filtración de acelerador 0,5 a $30 \mathrm{~Hz}$ desde los sectores occipitales $\left(\mathrm{O}_{1,2}\right)$, parietales $\left(\mathrm{P}_{3,4}\right)$, centrales $\left(\mathrm{C}_{3,4}\right)$, frontales $\left(F_{3,4}\right)$, temporales anteriores $\left(T_{3,4}\right)$ y temporales posteriores $\left(T_{5,6}\right)$, ubicados simétricamente de acuerdo con el esquema estándar 10-20\% encima del hemisferio derecho e izquierdo. El registro se realizó en estado de reposo (vigilia con ojos cerrados) y durante pruebas funcionales de hiperventilación y fotoestimulación rítmica. Para la fotoestimulación se utilizaron estímulos luminosos con intensidad equivalente a 0,1 J.; la fre- 
Delgado, et al. Rev. chil. neuropsicol. 6(2): 99-107, 2011

Tabla 1. Características de los niños estudiados, de acuerdo con la severidad del TCE, la edad, la escolaridad y el tiempo de evolución.

\begin{tabular}{|c|c|c|c|c|c|c|c|c|c|}
\hline Sujeto & Severidad & Edad & Escolaridad & Lateralidad & $\begin{array}{l}\text { Tiempo } \\
\text { (entre TCE y NP-EEG) }\end{array}$ & PLCNT & $\begin{array}{l}\text { INT. } \\
\text { Q. }\end{array}$ & CONV & $\begin{array}{l}\text { PERD. } \\
\text { CONC. }\end{array}$ \\
\hline Masculino (KEV) & Severo & 8.8 & 3을 Primaria & Diestro & 104 dias & $\mathrm{Si}$ & $\mathrm{Si}$ & No & $\mathrm{Si}$ \\
\hline Femenino (OSV) & Severo & 8.6 & 3으 Primaria & Diestra & 60 dias & $\mathrm{Si}$ & $\mathrm{Si}$ & No & $\mathrm{Si}$ \\
\hline Femenino (Lin) & Leve & 5.6 & 10 Primaria & Diestra & 102 dias & No & No & No & No \\
\hline Masculino (GUS) & Moderado & 5.1 & 3으 Preescolar & Diestro & 70 dias & $\mathrm{Si}$ & No & $\mathrm{Si}$ & $\mathrm{Si}$ \\
\hline Femenino (DAN) & Leve & 7.9 & 10 Primaria & Diestra & 137 dias & No & No & No & No \\
\hline Masculino (FCO) & Moderado & 7.1 & 10 Primaria & Diestro & 35 dias & No & No & No & No \\
\hline Femenino (NAN) & Severo & 7.1 & 10 Primaria & Diestra & 33 dias & No & No & No & $\mathrm{Si}$ \\
\hline Masculino (ALF) & Moderado & 7.2 & 20 Primaria & Diestro & 48 dias & $\mathrm{Si}$ & No & $\mathrm{Si}$ & $\mathrm{Si}$ \\
\hline
\end{tabular}

PLCNT (Policontusión), INT. Q. (Intervención Quirugica), CONV. (Crisis Convulsiva), PERD. CONC. (Pérdida de conciencia)

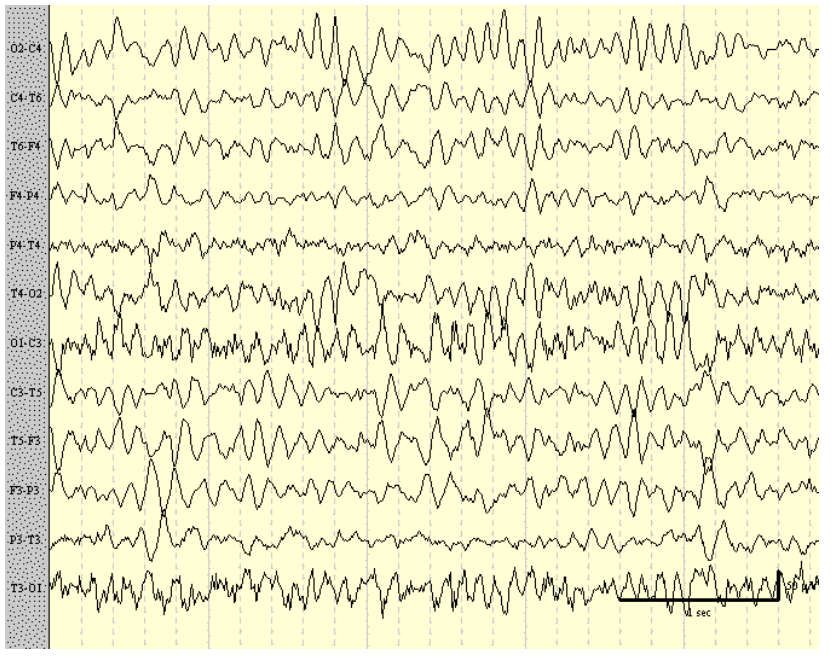

Figura 1. Ejemplo de registro EEG (bipolar) del paciente OSV. En este fragmento de EEG se observan ondas agudas de diapasón theta en fases de retorno en el registro frontal izquierdo (F3). La presencia de estas desviaciones locales en la actividad eléctrica señala cambios negativos en el estado funcional de las estructuras frontales del lóbulo frontal izquierdo. En el rincón derecho inferior - línea horizontal- la longitud del registro durante 1 segundo, línea vertical - amplitud de señal de EEG de 50 micro volts.

cuencia de fotoestimulación se cambiaba automáticamente de 4 hasta $12 \mathrm{~Hz}$ con paso en $1 \mathrm{HZ}$ ante la duración de la serie de estimulación de la misma frecuencia de 5-10 segundos con intervalo de 10 segundos entre las series.

\section{Procedimiento}

La muestra se seleccionó por muestreo no aleatorio en los departamentos de Neurocirugía y Pediatría del Hospital de Traumatología y Ortopedia del IMSS, en el que, por medio de entrevista a los padres, se verificaban los criterios de inclusión.

Posteriormente se aplicó la "Evaluación neuropsicológica infantil Puebla-Sevilla", de manera individual, con una duración de 90 minutos, en una o dos sesiones, dependien-

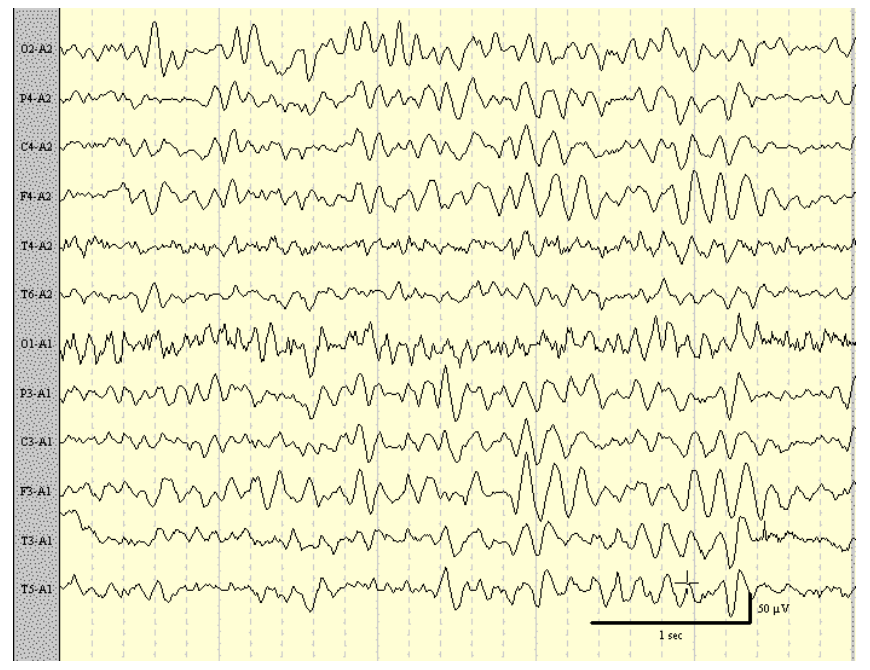

Figura 2. Ejemplo de registro EEG (monopolar) del paciente OSV. En los sectores frontales se observan oscilaciones bilaterales sincronizadas en forma de husos. Esto señala cambios funcionales desde las estructuras cerebrales límbicas.

do de las posibilidades de los niños. Durante la aplicación se registró el desempeño de cada uno de los niños, tales como la empatía con el evaluador, la orientación en persona, tiempo y lugar, cohesión y coherencia de su lenguaje, comportamiento en la situación de evaluación, muestra de interés para la ejecución de las tareas propuestas durante la sesión y actitud crítica hacia sus propias ejecuciones e intentos para corregirlas.

El registro EEG se realizó un mes después del TCE, en promedio. Cada especialista (neuropsicólogo, electrofisiólogo y neurólogo) realizó el análisis de sus resultados sin conocer los resultados de los otros estudios. Por ejemplo, el análisis cualitativo visual del EEG se realizó sin información acerca del carácter y la localización del TCE o del síndrome neuropsicológico resultante. La valoración de los patrones de EEG se realizó a partir del análisis cualitativo visual obtenido con montaje monopolar (con electrodos ipsilaterales del referente auricular) y bipolar. 
Tabla 2. Estructura de la Evaluación Neuropsicológica Infantil Puebla-Sevilla.

\begin{tabular}{|c|c|}
\hline Apartados & Tareas \\
\hline Programación y control & $\begin{array}{l}\text { Prueba verbal asociativa } \\
\text { Prueba verbal de conflicto }\end{array}$ \\
\hline Organización secuencial motora & $\begin{array}{l}\text { Copia y continuación de secuencia gráfica } \\
\text { Coordinación recíproca de las manos } \\
\text { Secuencia de movimientos manuales } \\
\text { Intercambio de posiciones de los dedos }\end{array}$ \\
\hline Integración cinestésica táctil & $\begin{array}{l}\text { Reproducción de posiciones de los dedos en la mano contraria } \\
\text { Evocación de posiciones de las manos } \\
\text { Reconocimiento de objetos } \\
\text { Reproducción de posiciones (aparato-fonoarticulario) } \\
\text { Repetición de sílabas y sonidos }\end{array}$ \\
\hline $\begin{array}{l}\text { Discriminación verbal-auditiva: integración fonemática (oído } \\
\text { fonemático) }\end{array}$ & $\begin{array}{l}\text { Repetición de pares de palabras } \\
\text { Repetición de sílabas } \\
\text { Identificación de fonemas: p-b; g-k (dar un golpe) } \\
\text { Reproducción de series de ritmos }\end{array}$ \\
\hline Retención audio-verbal y visual & $\begin{array}{l}\text { Evocación involuntaria: Repetición y evocación de dos series de palabras } \\
\text { Evocación voluntaria: Repetición se series de palabras. } \\
\text { Repetición de oraciones largas } \\
\text { Evocación de las series de palabras con interferencia heterogénea. } \\
\text { Memoria visual (copia y reproducción de letras; copia y reproducción de figuras no verbalizadas) } \\
\text { Reproducción de la serie de letras con la mano derecha (interferencia homogénea) } \\
\text { Reproducción de la serie de figuras con la mano izquierda (interferencia homogénea } \\
\text { Dibujo libre de una niña } \\
\text { Dibujo libre de un niño } \\
\text { Dibujo libre de } 4 \text { animales en cuadros marcados } \\
\text { Reproducción de los mismos } 4 \text { animales en los cuadros }\end{array}$ \\
\hline Percepción espacial Global & $\begin{array}{l}\text { Dibujo libre de una casa } \\
\text { Copia de una casa } \\
\text { Copia de letras con mano derecha } \\
\text { Copia de figuras con mano izquierda } \\
\text { Dibujo por consigna (mesa con } 4 \text { patas) } \\
\text { Dibujo en un reloj con manecillas } \\
\text { Dibujo de un reloj con las manecillas que muestren la hora 'cuarto para las 3' }\end{array}$ \\
\hline Percepción espacial analítica & $\begin{array}{l}\text { Completar figuras } \\
\text { Comprensión de oraciones con estructuras lógico-gramaticales complejas } \\
\text { Completar oraciones de acuerdo al cuadro } \\
\text { Comprensión de órdenes } \\
\text { Esquema corporal }\end{array}$ \\
\hline Atención y alerta & Identificación de estímulos significativos (figura-fondo) \\
\hline Esfera emocional & $\begin{array}{l}\text { Observaciones a partir de la entrevista a los familiares } \\
\text { Observaciones complementarias durante la sesión }\end{array}$ \\
\hline
\end{tabular}

Tabla 3. Resultados de la evaluación neuropsicológica, TAC y EEG en el paciente KEV, con TCE severo.

\begin{tabular}{|c|c|c|c|c|c|}
\hline TAC & $\begin{array}{c}\text { Evaluación } \\
\text { Neuropsicológica }\end{array}$ & $\begin{array}{c}\text { EEG } \\
\text { Estado de sistemas } \\
\text { ritmógenos corticales }\end{array}$ & $\begin{array}{c}\text { EEG } \\
\text { Ausencia/presencia de } \\
\text { cambios en el estado } \\
\text { cerebral general }\end{array}$ & $\begin{array}{c}\text { EEG } \\
\text { Patrones desviados en } \\
\text { sectores corticales }\end{array}$ & $\begin{array}{c}\text { EEG } \\
\text { Estado de estructuras } \\
\text { subcorticles }\end{array}$ \\
\hline $\begin{array}{l}\text { Hematoma epidural } \\
\text { bifrontal, laceración } \\
\text { dural frontobasal } \\
\text { izquierda. }\end{array}$ & $\begin{array}{l}\text { Inestabilidad general en } \\
\text { las ejecuciones, } \\
\text { impulsividad, } \\
\text { distrcatibilidad, } \\
\text { dificultades severas en la } \\
\text { retención visual ante } \\
\text { interferencia, } \\
\text { inversiones espaciales } \\
\text { en las tareas de } \\
\text { retención visual. }\end{array}$ & Sin cambios & No identificado & $\begin{array}{l}\text { Cambios en el estado } \\
\text { funcional de las zonas } \\
\text { parietal y temporal } \\
\text { anterior del HD de } \\
\text { origen subcortical. }\end{array}$ & $\begin{array}{l}\text { Cambios ligeros en el estado } \\
\text { funcional de las estructuras } \\
\text { hipotalámicas y meso y } \\
\text { diencefálicas. }\end{array}$ \\
\hline
\end{tabular}

Tabla 4. Resultados de la evaluación neuropsicológica, TAC y EEG en el paciente OSV, con TCE severo.

\begin{tabular}{|c|c|c|c|c|c|}
\hline TAC & $\begin{array}{c}\text { Evaluación } \\
\text { Neuropsicológica }\end{array}$ & $\begin{array}{l}\text { EEG } \\
\text { Estado de } \\
\text { sistemas } \\
\text { ritmógenos } \\
\text { corticales }\end{array}$ & $\begin{array}{c}\text { EEG } \\
\text { Ausencia/presencia de cambios } \\
\text { en el estado cerebral general }\end{array}$ & $\begin{array}{c}\text { EEG } \\
\text { Patrones desviados en } \\
\text { sectores corticales }\end{array}$ & $\begin{array}{c}\text { EEG } \\
\text { Estado de estructuras } \\
\text { subcorticles }\end{array}$ \\
\hline $\begin{array}{l}\text { Higromas frontales } \\
\text { bilaterales } \\
\text { residuales } \\
\text { laminares. }\end{array}$ & $\begin{array}{l}\text { Dificultades en la retención } \\
\text { audio-verbal en condiciones } \\
\text { de interferencia, en menor } \\
\text { grado en la retención visual, } \\
\text { dificultades en próximas } \\
\text { manuales de tipo cinestésico, } \\
\text { errores en la retención táctil. }\end{array}$ & Sin cambios & No identificado & $\begin{array}{l}\text { Cambios en el estado } \\
\text { funcional de los sectores } \\
\text { frontales del HI de } \\
\text { origen subcortical; } \\
\text { cambios menos notorios } \\
\text { en los sectores } \\
\text { simétricos del HD. }\end{array}$ & $\begin{array}{l}\text { Cambios ligeros en el estado } \\
\text { funcional de las estructuras } \\
\text { talámicas; cambios del estado } \\
\text { funcional de las estructuras } \\
\text { límbicas. }\end{array}$ \\
\hline
\end{tabular}


Delgado, et al. Rev. chil. neuropsicol. 6(2): 99-107, 2011

Tabla 5. Resultados de la evaluación neuropsicológica, TAC y EEG en el paciente LIN, con TCE leve.

\begin{tabular}{|c|c|c|c|c|c|}
\hline TAC & $\begin{array}{c}\text { Evaluación } \\
\text { Neuropsicológica }\end{array}$ & $\begin{array}{c}\text { EEG } \\
\text { Estado de } \\
\text { sistemas } \\
\text { ritmógenos } \\
\text { corticales }\end{array}$ & $\begin{array}{c}\text { EEG } \\
\text { Ausencia/presencia de } \\
\text { cambios en el estado } \\
\text { cerebral general }\end{array}$ & $\begin{array}{c}\text { EEG } \\
\text { Patrones desviados en } \\
\text { sectores corticales }\end{array}$ & $\begin{array}{c}\text { EEG } \\
\text { Estado de estructuras } \\
\text { subcorticles }\end{array}$ \\
\hline $\begin{array}{l}\text { Sin presencia de } \\
\text { hemorragias o } \\
\text { fracturas lineales, } \\
\text { línea media sin } \\
\text { desplazamiento, } \\
\text { ventrículos sin } \\
\text { desplazamiento, } \\
\text { edema cerebral. }\end{array}$ & $\begin{array}{l}\text { Problemas con la activación cerebral } \\
\text { general, alerta disminuida, } \\
\text { inestabilidad en las ejecuciones de las } \\
\text { tareas, rasgos de dismetría y } \\
\text { desproporción en las pruebas gráficas. } \\
\text { Inestabilidad en la organización } \\
\text { motora secuencial y en la regulación y } \\
\text { el control. }\end{array}$ & $\begin{array}{l}\text { Cambios en el } \\
\text { estado de los } \\
\text { sistemas } \\
\text { ritmógenos } \\
\text { corticales. }\end{array}$ & No identificado & $\begin{array}{l}\text { En el HD en general el } \\
\text { estado de la corteza se } \\
\text { encuentra alterado en } \\
\text { mayor grado en } \\
\text { comparación con el HI. En } \\
\text { HI predominan cambios } \\
\text { de origen profundo sin } \\
\text { localización precisa. }\end{array}$ & $\begin{array}{l}\text { Cambios severos del } \\
\text { estado funcional de las } \\
\text { estructuras del tronco } \\
\text { cerebral. }\end{array}$ \\
\hline
\end{tabular}

Tabla 6. Resultados de la evaluación neuropsicológica, TAC y EEG en el paciente GUS, con TCE moderado.

\begin{tabular}{|c|c|c|c|c|c|}
\hline TAC & $\begin{array}{c}\text { Evaluación } \\
\text { Neuropsicológica }\end{array}$ & $\begin{array}{c}\text { EEG } \\
\text { Estado de sistemas } \\
\text { ritmógenos corticales }\end{array}$ & $\begin{array}{c}\text { EEG } \\
\text { Ausencia/presencia de } \\
\text { cambios en el estado } \\
\text { cerebral general }\end{array}$ & $\begin{array}{c}\text { EEG } \\
\text { Patrones desviados en } \\
\text { sectores corticales }\end{array}$ & $\begin{array}{c}\text { EEG } \\
\text { Estado de estructuras } \\
\text { subcorticles }\end{array}$ \\
\hline $\begin{array}{l}\text { Hematoma epidural temporo } \\
\text { parietal derecha, fractura sin } \\
\text { desplazamiento. Contusion } \\
\text { temporal derecha, trazo de } \\
\text { fractura lineal adyacente, } \\
\text { hemorragia subaracnoidea a } \\
\text { nivel de la cisura } \\
\text { interhemisferica en región } \\
\text { occipital. }\end{array}$ & $\begin{array}{l}\text { Leves dificultades en } \\
\text { regulación y control, } \\
\text { inestabilidad en la ejecución } \\
\text { de las tareas, cierta } \\
\text { dismetría y desproporción a } \\
\text { nivel perceptivo gráfico. }\end{array}$ & $\begin{array}{l}\text { Cambios en el estado } \\
\text { de los sistemas } \\
\text { ritmógenos corticales } \\
\text { en el HD; en HI se } \\
\text { observa excitación } \\
\text { cotical elevada. }\end{array}$ & No identificado & $\begin{array}{l}\text { Cambios severos en el } \\
\text { estado funcional de los } \\
\text { sectores temporales y } \\
\text { parietales del HD de } \\
\text { origen profundo; } \\
\text { cambios secundarios } \\
\text { en el HI. }\end{array}$ & $\begin{array}{l}\text { Cambios severos del } \\
\text { estado funcional de las } \\
\text { estructuras del tronco } \\
\text { cerebral. }\end{array}$ \\
\hline
\end{tabular}

Tabla 7. Resultados de la evaluación neuropsicológica, TAC y EEG en el paciente DAN, con TCE leve.

\begin{tabular}{|c|c|c|c|c|c|}
\hline TAC & $\begin{array}{l}\text { Evaluación } \\
\text { Neuropsicológica }\end{array}$ & $\begin{array}{c}\text { EEG } \\
\text { Estado de } \\
\text { sistemas } \\
\text { ritmógenos } \\
\text { corticales }\end{array}$ & $\begin{array}{c}\text { EEG } \\
\text { Ausencia/presencia de } \\
\text { cambios en el estado } \\
\text { cerebral general }\end{array}$ & $\begin{array}{c}\text { EEG } \\
\text { Patrones desviados en } \\
\text { sectores corticales }\end{array}$ & $\begin{array}{l}\text { EEG } \\
\text { Estado de estructuras subcorticles }\end{array}$ \\
\hline $\begin{array}{l}\text { Hematoma subgaleal } \\
\text { extenso, fronto parietal } \\
\text { izquierdo, fractura } \\
\text { lineal parietal } \\
\text { izquierda, edema } \\
\text { cerebral. }\end{array}$ & Ejecuciones correctas & Sin cambios & No identificado & No identificado & $\begin{array}{l}\text { Cambios severos en el estado } \\
\text { funcional de las estructuras } \\
\text { hipotalámicas de carácter } \\
\text { paroxístico; cambios severos en el } \\
\text { estado funcional de las estructuras } \\
\text { del tronco superior, de predominio } \\
\text { en } \mathrm{HI} \text {. }\end{array}$ \\
\hline
\end{tabular}

Tabla 8. Resultados de la evaluación neuropsicológica, TAC y EEG en el paciente FCO, con TCE moderado.

\begin{tabular}{|c|c|c|c|c|c|}
\hline TAC & $\begin{array}{c}\text { Evaluación } \\
\text { Neuropsicológica }\end{array}$ & $\begin{array}{c}\text { EEG } \\
\text { Estado de sistemas } \\
\text { ritmógenos corticales }\end{array}$ & $\begin{array}{c}\text { EEG } \\
\text { Ausencia/presencia de } \\
\text { cambios en el estado } \\
\text { cerebral general }\end{array}$ & $\begin{array}{c}\text { EEG } \\
\text { Patrones desviados en } \\
\text { sectores corticales }\end{array}$ & $\begin{array}{c}\text { EEG } \\
\text { Estado de estructuras } \\
\text { subcorticles }\end{array}$ \\
\hline $\begin{array}{l}\text { Fractura sin } \\
\text { desplazamiento en región } \\
\text { frontal, sin lesión en canal } \\
\text { auditivo, fractura de piso } \\
\text { medio izquierdo, no hay } \\
\text { edema. }\end{array}$ & $\begin{array}{l}\text { Severas alteraciones } \\
\text { de regulación y } \\
\text { control pérdida del } \\
\text { objetivo en las } \\
\text { tareas, } \\
\text { perseveraciones } \\
\text { severas. }\end{array}$ & $\begin{array}{l}\text { En general no se han } \\
\text { identificado cambios } \\
\text { notoios en los sistem } \\
\text { ritmógenos corticales, se } \\
\text { observa cierta disminución } \\
\text { de la frecuencia de ritmo } \\
\text { de fondo. }\end{array}$ & No identificado & $\begin{array}{l}\text { Cambios en el estado } \\
\text { funcional del sector } \\
\text { parieto-temporal de } \mathrm{HI} \\
\text { de origen profundo. }\end{array}$ & $\begin{array}{l}\text { Cambios en el estado } \\
\text { funcional de las estructuras } \\
\text { del tronco superior. }\end{array}$ \\
\hline
\end{tabular}

Tabla 9. Resultados de la evaluación neuropsicológica, TAC y EEG en el paciente NAN, con TCE severo.

\begin{tabular}{|c|c|c|c|c|c|}
\hline TAC & $\begin{array}{c}\text { Evaluación } \\
\text { Neuropsicológica }\end{array}$ & $\begin{array}{c}\text { EEG } \\
\text { Estado de sistemas } \\
\text { ritmógenos corticales }\end{array}$ & $\begin{array}{c}\text { EEG } \\
\text { Ausencia/presencia de } \\
\text { cambios en el estado } \\
\text { cerebral general }\end{array}$ & $\begin{array}{c}\text { EEG } \\
\text { Patrones desviados en sectores } \\
\text { corticales }\end{array}$ & $\begin{array}{c}\text { EEG } \\
\text { Estado de estructuras } \\
\text { subcorticles }\end{array}$ \\
\hline $\begin{array}{l}\text { Primera TAC edema } \\
\text { cerebral. Segunda } \\
\text { TAC muestra } \\
\text { normalidad. }\end{array}$ & $\begin{array}{l}\text { Ligeras dificultades } \\
\text { en regulación y } \\
\text { control, disminución } \\
\text { severa de la } \\
\text { retención visual y } \\
\text { audio-verbal ante } \\
\text { interferencia. }\end{array}$ & Sin cambios & No identificado & $\begin{array}{l}\text { Cambios expresivos en el estado } \\
\text { funcional del sector central parieto } \\
\text { occipital de HI de origen profundo; } \\
\text { cambios locales de la actividad } \\
\text { eléctrica en el sector frontal de HD; } \\
\text { cambios locales se incrementan hasta } \\
\text { descargas paroxísticas durante } \\
\text { hiperventilación. }\end{array}$ & No identificado \\
\hline
\end{tabular}


Tabla 10. Resultados de la evaluación neuropsicológica, TAC y EEG en el paciente ALF, con TCE moderado.

\begin{tabular}{|c|c|c|c|c|c|}
\hline TAC & $\begin{array}{c}\text { Evaluación } \\
\text { Neuropsicológica }\end{array}$ & $\begin{array}{c}\text { EEG } \\
\text { Estado de sistemas } \\
\text { ritmógenos corticales }\end{array}$ & $\begin{array}{c}\text { EEG } \\
\text { Ausencia/presencia de } \\
\text { cambios en el estado } \\
\text { cerebral general }\end{array}$ & $\begin{array}{c}\text { EEG } \\
\text { Patrones desviados en } \\
\text { sectores corticales }\end{array}$ & $\begin{array}{c}\text { EEG } \\
\text { Estado de estructuras } \\
\text { subcorticles }\end{array}$ \\
\hline $\begin{array}{l}\text { Normal, si presencia } \\
\text { de edema, } \\
\text { colecciones sin } \\
\text { sangrado, se } \\
\text { descarta lesión } \\
\text { intracraneal. }\end{array}$ & $\begin{array}{l}\text { Dificultades en la } \\
\text { organización motora } \\
\text { secuencial, sustituciones } \\
\text { cinestésicas en las tareas } \\
\text { de repetición y evocación, } \\
\text { dificultades en todas las } \\
\text { pruebas motoras. }\end{array}$ & Sin cambios expresivos & No identificado & $\begin{array}{l}\text { Cambios en el estado } \\
\text { funcional de } \mathrm{HI} \text { en los } \\
\text { sectores frontal, central } \\
\text { y temporal posterior de } \\
\text { origen profundo. }\end{array}$ & $\begin{array}{l}\text { Cambios en el estado } \\
\text { funcional en las estructuras } \\
\text { hipotalámicas, límbicas y del } \\
\text { tronco superior. }\end{array}$ \\
\hline
\end{tabular}

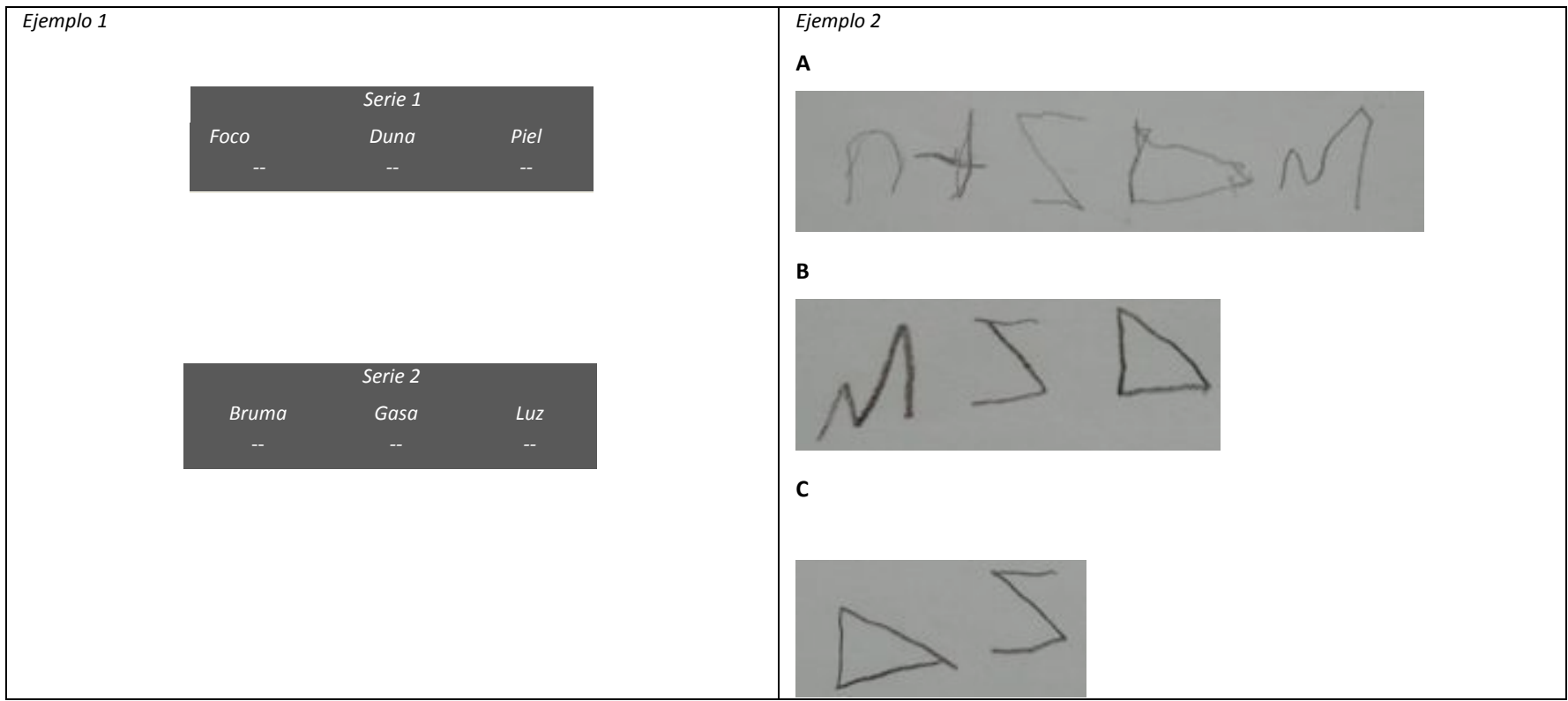

Figura 3. Ejemplo 1. Ejecución del paciente OSV en la tarea de retención audioverbal (evocación libre ante interferencia). El paciente no logra recordar ninguna de las palabras. Ejemplo 2. Ejecución del paciente OSV en la tarea de retención visual: (A) copia, (B) reproducción inmediata y (C) evocación ante interferencia.

\section{Resultados}

Los resultados de la evaluación neuropsicológica se correlacionaron con los resultados de la TAC y del EEG en cada uno de los casos.

En el caso de paciente KEV (ver tabla 3), se observa una correspondencia entre las dificultades espaciales gráficas con el registro EEG, caracterizados por cambios funcionales de la región parietal del hemisferio derecho. Lo anterior concuerda con estudios experimentales realizados con niños que reportan la participación de regiones parietales en el procesamiento de la información espacial (Wilson, 1988; Willis \& Weiler, 2005; Solovieva \& cols., 2009). Las dificultades en la retención visual ante interferencia se correlacionan con los cambios funcionales de los sectores temporales, debido a que en el EEG se refleja el estado de las estructuras del hipocampo y de las estructuras subcorticales profundas (meso y diencefálicas).

En el caso del paciente OSV (tabla 4) se identificaron cambios importantes en el funcionamiento del sector frontal del hemisferio izquierdo, así como en las estructuras del sistema límbico (figuras 1 y 2). Estos últimos cambios podrían correlacionarse con las dificultades en los procesos de retención audio verbal y visual (ejemplos 1 y 2 ).

En el paciente LIN (tabla 5) se observa la presencia de cambios funcionales en las estructuras del tronco cerebral, lo cual se relaciona con el bajo nivel de activación general e inestabilidad que el paciente mostró durante la ejecución de la mayoría de las tareas de la evaluación neuropsicológica. Asimismo, se observa mayor presencia de patrones desviados de la actividad eléctrica en el hemisferio derecho, en comparación con el hemisferio izquierdo, lo cual se correlaciona con la dismetría y la desproporción en las tareas gráficas.

En el paciente GUS (tabla 6) el EEG reveló cambios severos en las estructuras del tronco cerebral, que se manifiestan en inestabilidad del nivel de atención y procesos de regulación de la actividad. Por otro lado, los cambios funcionales severos en las regiones témporo-parietales del HD se pueden correlacionar con las dificultades en las tareas espaciales.

En el caso del paciente DAN (tabla 7) no se observaron correlaciones entre los resultados. No obstante que el EEG muestra un estado funcional desfavorable de las estructuras del tronco superior, el paciente realizó todas las tareas de la evaluación neuropsicológica de manera correcta.

En el caso del paciente FCO (tabla 8), el EEG reveló signos desfavorables en el estado funcional de las estructuras del tronco superior (regulación fronto-talámica). Además, se observó un estado funcional desfavorable del hemisferio izquierdo, con cambios en los sistemas ritmógenos esperados para su edad (7,1 años), lo cual indica un bajo nivel de madurez. Dichos cambios funcionales se correlacionan con la presencia de perseveraciones, pérdida del objetivo y con severas dificultades 
para la regulación y el control de la actividad durante toda la valoración neuropsicológica.

En el caso del paciente NAN (tabla 9), el EEG reveló cambios funcionales en los sectores parieto-occipitales del hemisferio izquierdo y en sectores frontales del hemisferio derecho. Estos cambios se correlacionan con las dificultades en las tareas de retención visual y audio-verbal, así como con las dificultades leves para regular y controlar la actividad.

En el caso del paciente ALF (tabla 10), el EEG muestra cambios funcionales en los sectores frontal y central del hemisferio izquierdo, así como en las estructuras del tronco superior. Dichos cambios se correlacionan con las dificultades para la organización secuencial motora y con la presencia de sustituciones de sonidos por punto y modo de articulación.

\section{Discusión}

Los resultados de la evaluación neuropsicológica revelaron cuadros clínicos diferenciales en los niños escolares con TCE estudiados. Sin embargo, las dificultades registradas no se correlacionan con el grado de severidad de TCE en todos los pacientes. Algunos estudios (Alexander, 1997; Taryn \& cols., 2010) señalan que el desempeño de los pacientes no depende necesariamente del grado de severidad del TCE, sino que puede estar relacionado con factores como las características premorbidas, el nivel de éxito escolar, el nivel educativo de los padres, etc.

Los estudios de gabinete, como la TAC, permiten identificar los sectores cerebrales afectados en los casos de TCE. Sin embargo, la localización de la lesión no garantiza la comprensión del cuadro clínico, debido a que no puede explicar la forma en que los procesos psicológicos pueden afectarse como resultado de la desorganización funcional de los diversos sistemas cerebrales que produce un TCE.

En nuestro estudio, los resultados de la TAC y de la evaluación neuropsicológica solo se correlacionaron en los casos KEV y GUS, y en menor medida en el caso FCO. En tres casos (LIN, NAN y ALF), la TAC se reporta como normal, pero la evaluación neuropsicológica sí reporta alteraciones. En el caso OSV las alteraciones neuropsicológicas no se correlacionan con la lesión que reporta la TAC. Finalmente, en el caso DAN no se observaron alteraciones neuropsicológicas, a pesar de que la TAC sí reporta afectación. Resultados similares fueron reportados por Maillard-Wermeinger y cols. (2009), quienes observaron anomalías en los estudios estructurales de resonancia magnética solo en $18 \%$ de los 186 casos estudiados. Nuestros resultados muestran correlaciones interesantes entre la actividad eléctrica cerebral y los resultados de la evaluación neuropsicológica. En general, los resultados del análisis del EEG mostraron estados funcionales desfavorables en diversas estructuras cerebrales en todos los niños con TCE estudiados. Prácticamente en todos los niños se registraron desviaciones locales funcionales en distintas zonas corticales, además de ondas agudas en grupos de oscilaciones lentas de amplitud alta.

En cuatro de los casos (KEV, OSV, LIN y GUS), el EEG reporta un estado funcional desfavorable de estructuras cerebrales profundas (tronco inferior), que clínicamente se correlaciona con inestabilidad en las ejecuciones y problemas en la recuperación de la información en diversas modalidades durante la valoración neuropsicológica. Además de lo anterior, en los casos KEV, LIN y GUS se observaron dificultades en el procesamiento de la información visuo-espacial, problemas con la representación de la imagen objetal, dismetría y desproporción en la actividad gráfica, que se correlacionan con el estado funcional desfavorable en el hemisferio derecho registrado en tales casos.

Es interesante observar que en el caso DAN, a pesar de que la TAC reporta fractura lineal parietal izquierda y edema, no se presentaron dificultades en la valoración neuropsicológica y el EEG no reporta signos de patrones desviados de la actividad eléctrica cortical, solo subcortical. Por su parte, las dificultades severas en la programación y el control (caso FCO) y las alteraciones en la organización secuencial motora (caso ALF) se correlacionan con el registro EEG, que reporta un estado funcional desfavorable del tronco superior y de los sectores frontales y centrales del hemisferio izquierdo.

Particularmente en el caso FCO, en el que se observaron serias dificultades para la regulación y el control, el EEG muestra rasgos de inmadurez del sistema de regulación fronto-talámica (tronco superior), lo cual probablemente se relaciona con las características propias del niño, ajenas al trauma cerebral. Es importante señalar que durante el desarrollo se debe diferenciar entre las consecuencias propias del TCE y el estado de inmadurez premórbido de estructuras cerebrales particulares (Alexander, 1997; Yeats \& Taylor, 2005). En el caso FCO se puede hipotetizar que la combinación del estado de inmadurez del sistema de regulación fronto-talámica con el traumatismo produce efectos particularmente severos, que se manifiestan en los errores y en la imposibilidad para acceder a las tareas de la evaluación neuropsicológica. En otros estudios (Solovieva y cols., 2008, 2009) se reporta que el estado de inmadurez de la regulación fronto-talámica está presente en la mayoría de los niños diagnosticados con trastorno por déficit de atención.

Lo anterior permite suponer que, en los casos de TCE, los cambios en el estado funcional de las redes neuronales corticales se deben al decremento de la llegada de señales a la corteza cerebral, como consecuencia de alteraciones del funcionamiento de la sustancia blanca (vías conductoras córtico-corticales y córtico-subcorticales). Esto se basa en los reportes acerca de los mecanismos de generación de oscilaciones lentas en la corteza cerebral (Steriade y cols., 1990).

Otra causa probable de los cambios en el estado funcional de sectores corticales específicos después de un TCE puede ser el decremento de la circulación sanguínea local (Korn \& cols., 2005). En nuestro estudio se registraron cambios locales (no difusos) de la actividad eléctrica cerebral en forma de incremento de la expresividad de oscilaciones lentas en el EEG, cuya fuente se encuentra en la corteza cerebral. La TAC no reveló cambios morfológicos directamente en el sitio de la activación desviada en la corteza cerebral. Es probable que ambos mecanismos hipotéticos, el decremento de la conducción de los impulsos en la sustancia blanca y de la circulación sanguínea local en la corteza, estén relacionados entre sí.

Otra particularidad de los cambios locales registrados en el EEG de los niños estudiados con TCE es que incluían varias zonas corticales cercanas del mismo hemisferio, lo cual puede ser la causa del complejo de alteraciones de los procesos cognitivos en cada caso particular. Es interesante que, en algunos casos, la topografía de los cambios del estado funcional 
cerebral no coincide, o no completamente, con la localización del trauma, como en los casos KEV, OSV, LIN y NAN. Podemos decir que, en algunos casos, el registro EEG proporciona información complementaria útil acerca de las bases cerebrales de los síndromes neurológicos y neuropsicológicos que surgen como consecuencia de TCE.

Para la comprensión de las causas de las alteraciones neurológicas y cognitivas en niños escolares con TCE resultan de gran interés los cambios expresivos del estado de las estructuras subcorticales reguladoras de diversos niveles, registrados en todos los niños analizados, con excepción del caso NAN. Estos cambios pueden producir efectos específicos sobre el estado funcional de diversos componentes atencionales, en dependencia de su localización en el tronco cerebral (Machinskaya y Semenova, 2004).

Respecto de la severidad del trauma, es interesante que en los casos KEV, OSV y NAN, con TCE severo, en los cuales se esperarían alteraciones importantes de los procesos cognitivos (Hanten, 2002; Ylvisaker, 1998; Bigler, 1990), particularmente de aquellas funciones relacionadas con las regiones frontales, como atención y funciones ejecutivas (Mangeot, 2002), no se presentaron dificultades tan severas durante la valoración neuropsicológica. Solo en el caso KEV se observó una alteración de mayor importancia en los niveles de atención sostenida, así como muestras significativas de impulsividad.

Por el contrario, los casos FCO y ALF, diagnosticados con TCE moderado, presentaron dificultades más severas durante la valoración neuropsicológica, en comparación con el caso NAN con TCE severo. En los dos primeros casos el EEG registró patrones eléctricos desviados de mayor importancia.

De igual manera, ante la comparación de los pacientes 3 y 4 , diagnosticados como TCE leve y moderado respectivamente, es posible identificar las limitaciones considerablemente mayores en el caso de la paciente 3 , mostrando errores que no pueden ser explicados como parte del desarrollo normal para su rango de edad (Loredo, 2008).

Podemos concluir que el análisis neuropsicológico y electrofisiológico arroja información útil para la comprensión de las manifestaciones clínicas de niños con TCE. Esta información no necesariamente se encuentra en una relación directa con los datos que sugiere TAC ni con el grado de severidad del TCE en el niño, lo cual coincide con los hallazgos de Maillard-Wermelinger y cols. (2009).

\section{Conclusiones}

Nuestros resultados muestran que el grado y la extensión del daño cerebral no es un parámetro estricto para determinar el nivel de afectación de las funciones cognitivas en los casos de TCE. Por otro lado, el grado de severidad del TCE inicial, o durante su evolución inicial, no determina las secuelas neuropsicológicas.

Los resultados indican que la evaluación neuropsicológica puede ser de gran utilidad para caracterizar el cuadro clínico y las dificultades específicas que presentan los niños escolares como consecuencia de TCE.

El análisis cualitativo visual del EEG ofrece información fundamental acerca de los cambios en el funcionamiento de los sistemas cerebrales en los casos de TCE.
Los resultados de la valoración neuropsicológica no se correlacionan $100 \%$ con la localización del daño que reporta la TAC, pero mantienen una correlación directa con los resultados del registro EEG.

La caracterización clínica y la identificación del estado funcional de los diferentes sistemas cerebrales constituyen la premisa para la elaboración del programa de rehabilitación que garantizará la recuperación del paciente.

\section{Agradecimientos}

Al CONACYT por la beca otorgada a Abel Armando Delgado Mota (becario № 226594) para realizar estudios de maestría. A la Vicerrectoría de Investigación y Estudios de Posgrado de la Universidad Autónoma de Puebla, por los recursos otorgados al proyecto "Correlación neuropsicológica y electrofisiológica en niños escolares con TCE".

\section{Referencias}

Alexander, M. P. (1997). Minor traumatic brain injury: A review of psychiogenesis and psychogenesis. Seminars in Clinical Neuropsychiatry, 2, 177-187.

Anderson, V. \& Yeates, K. O. (2010). Pediatric Traumatic Brain Injury. New frontiers in clinical and traslational research. Estados Unidos: Cambridge University Press.

Ariza, M., Pueyo, R., \& María, S. G. (2004). Secuelas neuropsicológicas de los traumatismos craneoencefálicos. Anales de Psicología, 20(2), 303-316.

Ashley, M. \& Krych, D. (1995). Traumatic Brain Injury Rehabilitation. Estados Unidos: CRC Press.

Bejarano, L., Ramírez, D. y Ramírez, M.M. (2008). Traumatismo Craneoencefálico en niños: Relación entre los hallazgos tomográficos y el pronóstico. Revista de Especialidades Médico-Quirúrgicas, 13(2), 60-68.

Bezrukikh, M. M., Machinkaya, R.I. \& Sugrobova, G.A. (1999). Differentiated Influence of the functional maturity of the Cortex and Brain regulatory structures on the characteristics of cognitive activity in 7-8 year old Children. Human Physiology, 24(5), 510-517.

Bigler, E. (1990). Traumatic Brain Injury. Estados Unidos: Pro-ed.

Christensen, A. \& Uzzell, B. (1994). Brain Injury and Neuropsychologycal Rehabilitation, International Perspectives. Estados Unidos: Lawrence Erlbaum Associates Publishers.

Dougherty, D., Rauch, S. \& Rosenbaum, J. (2004). Essentials of Neuroimaging for Clinical Practice. Estados Unidos: American Psychiatric Publishing, Inc.

Garduño, F. (2000). Traumatismo Craneoencefálico en niños. Mecanismos de lesión, restauración cerebral y prevención. Bol. Med. Hospital Infantil México, $57(2), 342-350$.

Hanten, G., Zhang, L. \& Levin, H. (2002). Selective Learning in Children after Traumatic Brain Injury: A Preliminary Study. Child Neuropsychology, 8(2), 107-120.

Hughes, J. R. (1994). EEG in Clinical Practice. Second Edition. Boston: ButterworthHeinemann.

Jiménez, S, (2009). Características neuropsicológicas de escolares entre 8 y 12 años de edad con Traumatismo Craneoencefálico. México: Benemérita Universidad Autónoma de Puebla (inédito).

Kaye, A. (2005). Essential Neurosurgery. Estados Unidos: Blackwell Publishing Ltd.

Korn, A., Golan, H., Melamed, I., Pascual-Marqui, R. \& Friedman, A. (2005). Focal cortical dysfunction and blood-brain barrier disruption in patients with postconcussion syndrome. J Clin Neurophysiol, 22, 1-9.

Lacerda-Gallardo, A. J. y Abreu-Pérez, D. (2003). Traumatismo Craneoencefálico en Pediatría. Nuestros Resultados. Revista de Neurología, 36(2), 108-112.

Long, C. \& Ross, L. (1992). Handbook of Head Trauma. Nueva York: Plenum Press

Loredo, D. (2008). Caracterización Neuropsicológica de una población infantil urbana. México: Benemérita Universidad Autónoma de Puebla (inédito).

Lukashevich I. P., Machinskaya R. I \& Fishman M. N. (1999). The EEG-Expert Automatic Diagnostic System. Biomedical Engineering, 33(6), 302-307.

Luria, A. R. (1969). Las funciones corticales superiores del hombre. Moscú: Universidad Estatal de Moscú.

Luria, A. R. (1979). El cerebro en acción. Barcelona: Ed. Fontanella.

Machinskaya, R. I., Lukashevich, I. P., \& Fishman M. N. (1997). Dynamics of Brain Electrical Activity in 5- to 8-Year-old normal children and children with Learning Difficulties. Human Physiology, 23(5), 517-522.

Machinskaya, R. I., Sokolova, L. S. y Krupskaya, E. V. (2007). Formation of the Functional Organization of the Cerebral Cortex at rest in young schoolchildren varying in the maturity of Cerebral Regulatory Systems: II. Analysis of EEG Alpha Rhythm Coherence. Human Physyology, 33(2), 129-138. 
Machinskaya, R. I., y Semenova, O. A. (2004). Peculiarities of formation of the cognitive functions in junior school children with different maturity of regulatory brain systems. Journal of Evolutionary Biochemistry and Physiology, 40(5), 528-538.

Maillard-Wermelinger, A., Yeates, K. O., Taylor, H. G., Rusin, J., Bangert, B., Dietrich, A., Nuss, K. \& Wrigth, M. (2009). Mild traumatic brain injury and ejecutive functions in school-aged children. Devevolmental Neurorehabilitation, 12(5), 330-341.

Mangeot, S., Armstrong, K., Colvin, A., Yeates, K. \& Taylor, H. (2002). Long term Executive Function deficit in children with Traumatic Brain Injuries assesment using the Behavior Rating Inventory of Executive Function (BRIEF). Child Neuropsychology, 8(4), 271-284.

McCRea, M. (2008). Mild Traumatic Brain Injury and Postconcussion Syndrome. Estados Unidos: Oxford Unoversity Press.

Mundy, P., Fox, N. \& Card, J. (2003). EEG Coherence, Joint Attention and language development in the second year. Developmental Science, 6(1), 48-55.

Muñóz-Céspedes, J. M., Paúl-Lapedriza, N., Pelegrín-Valero, C. \& Tirapú-Ustarroz, J. (2001). Factores de pronóstico en los Traumatismos Craneoencefálicos. Revista de Neurología, 32(4), 351-364.

Murillo, M. (2008). Traumatismo Craneoencefálico del Niño y del Adolescente. México: McGraw Hill.

Naunheim, R., Treaster, M., English, J., Casner, T. \& Chabot, R. (2010). Use of Brain Electrical Activity to quantify traumatic brain injury in the emergency department. Brain Injury, 24(11), 1324-1329.

Parker, R. (2001). Concussive Brain Trauma. Estados Unidos: CRC Press.

Pérez, M. F. (2010). Exploración Neuropsicológica, Electrofisiológica y de Neuroimagen Estructural en niños con traumatismo craneoencefálico severo. México: Benemérita Universidad Autónoma de Puebla (inédito).

Pivik, R., Broughton, R., Coppola, R., Davidson, R., Fox, N. \& Nuwer, M. (1993). Guidelines for the recording and quantitative Analysis of Electroencephalographic Activity in research contexts. Psychophysiology, 30, 547-558.

Quintanar, L., \& Solovieva, Y. (2003). Manual de evaluación neuropsicológica infantil. Puebla, México: Benemérita Universidad Autónoma de Puebla.

Quintanar, L. \& Solovieva, Yu. (2008). Aproximación histórico-cultural: fundamentos teórico-metodológicos. En: Eslava-Cobos J., Mejía L., Quintanar L. y Solovieva Yu. Los trastornos de aprendizaje: perspectivas neuropsicologías. Textos de neuropsicología Latinoamericana. Tomo 1. Colombia, Magisterio: 145-182.

Saunders M. G. \& Westmoreland B. F. (1979). The EEG in evaluation of disorders affecting the brain diffusely. In Klass D.W., Daly D.D. eds. Current Practice of Clinical Electroencephalography. New-York: Raven Press.

Secretaría de Salud, Sistema Nacional de Vigilancia Epidemiológica (2008). Aspectos Clínicos y Epidemiológicos del Trauma Cráneo Encefálico en México. Vigilancia Epidemiológica, 25(26), 1-4.

Shaw, J. (comp.) (2003). The brain alpha rhythms and the Mind. Netherland: Elsevier.

Sistema Nacional de Salud, Consejo de Salubridad General (2008). Atención Inicial del Traumatismo Craneoencefálico en pacientes menores de 18 Años. Guía de Práctica Clínica GPC. Catalogo Maestro de Guías de Práctica Clínica SSA002-08.

Solovieva, Yu. \& Quintanar, L. (2009). Evaluación neuropsicológica infantil breve. México: Universidad Autónoma de Puebla.

Solovieva, Y., Machinskaya, R., Quintanar, L., Bonilla, M.R. \& Pelayo, H. (2009). Neuropsicología y Electrofisiología del TDA en la edad Preescolar. México: Benemérita Universidad Autónoma de Puebla.

Steriade, M., Gloor, P., Llinas, R. R., Lopes da Silva, F. H. \& Mesulam, M. M. (1990) Report of IFCN Committee on basic mechanisms: Basic mechanisms of Cerebral rhythmic activities. Electroencephalography and Clinical Neurophysiology, 76, 481-508.

Stoccheti, N., Conte, V., Ghisoni, L., Canavesi, K. \& Zanaboni, C. (2010). Traumatic Brain Injury in Pediatric Patients. Minerva Anestesiológica, 76, 1052-1059.

Taryn B. F., Yeats, K, O., Taylor, G. H., Bangert, B. A., Nuss, D. K., Rusin, J. \& Wright, M. (2010). Cognitive reserve as a moderator of postconcussive symptoms in children with complicated and uncomplicated mild traumatic brain injury. Journal of International Neuropsychological Society, 16(1), 94-105.

Thatcher, R. W., Biver, C., Gomez, J., North, D., Curtin, R., Walker, R. \& Salazar, A. (2001a). Estimation of the EEG Power Spectrum using MRI T2 Relaxation time in Traumatic Brain Injury. Clinical Neurophysiology, 112, 1729-1745.

Thatcher, R. W., North, D., Curtin, R., Walker, R., Biver, C., Gomez, J. \& Salazar, A. (2001b). An EEG Severity Index of Traumatic Brain Injury. J. Neuropsychiatry Clin Neurosci, 13(1), 77-87.

Willis, W. G., Weiler, M. D. (2005). Neural substrates of childhood attention-deficit hyperactivity disorder: Electroencephalographic and magnetic resonance imaging evidence. Developmental Neuropsychology, 27,135-82.

Wilson, H. K. (1988). Development of spatiotemporal mechanisms of infant vision. Vision Research, 28, 611-628.

Wardlaw, J. M., Easton, V. J. \& Statham, P. (2002). Which CT features help predict outcome after Head Injury? J Neurol Neurosurg Psychiatry, 72, 188-192.
Yeats, K. O., \& Taylor, H. G. (2005). Neurobehavioral outcomes of mild head injury in children and adolescents. Pediatric Rehabilitacion, 8(1), 5-16.

Ylvisaker, M. (1998). Traumatic Brain Injury Rehabilitation. Estados Unidos: Butterworth-Heinemann. 